\title{
RANK 2 VECTOR BUNDLES ON HIGHER DIMENSIONAL PROJECTIVE MANIFOLDS
}

\author{
E. BALLICO
}

Fix a smooth complex projective $n$-fold $X, n \geq 5$, with $H^{1}\left(X, \mathcal{O}_{X}\right)=0$. Then there is a smooth projective $n$-fold $Y$ birational to $X$ and $H \in \operatorname{Pic}(Y), H$ ample, with the following property. For any $d \in \mathbf{Z}$ let $M(Y, 2, \mathcal{O}, d, H)$ be the moduli scheme of $H$-stable rank 2 vector bundles, $E$, on $Y$ with $\operatorname{det}(E) \cong \mathcal{O}_{Y}$ and $c_{2}(E) \cdot H^{n-2}=d$. Let $m(Y, 2, \mathcal{O}, d, H)$ be the number of its irreducible components. Then $\lim \sup _{d \rightarrow \infty} m(Y, 2, \mathcal{O}, d$, $H)=+\infty$.

Usually it is very hard to construct vector bundles of low rank on a projective manifold $X$ of large dimension. Among the vector bundles, by far the more interesting ones are the stable ones. Here we will show that if we take a suitable birational model $Y$ of $X$, then the construction is "easy" in the following sense.

Theorem 0.1. Assume characteristic 0. Fix a smooth projective n-fold $X, n \geq 5$, with $H^{1}\left(X, \mathcal{O}_{X}\right)=0$. Then there is a smooth projective $n$-fold $Y$ birational to $X$ and $H \in \operatorname{Pic}(Y), H$ ample, with the following property. For any $d \in \mathbf{Z}$ let $M(Y, 2, \mathcal{O}, d, H)$ be the moduli scheme of H-stable rank 2 vector bundles, $E$, on $Y$ with $\operatorname{det}(E) \cong \mathcal{O}_{Y}$ and $c_{2}(E) \cdot H^{n-2}=$ d. Let $m(Y, 2, \mathcal{O}, d, H)$ be the number of its irreducible components. Then $\lim \sup _{d \rightarrow \infty} m(Y, 2, \mathcal{O}, d, H)=+\infty$.

Here, $H$-stability is in the sense of Mumford-Takemoto.

Remark 0.2. $\quad M(Y, 2, \mathcal{O}, d, H)$ is an algebraic scheme and not only a locally algebraic scheme because the vector bundles in $M(Y, 2, \mathcal{O}, d, H)$ may have only finitely many Hilbert polynomials with respect to $H$. Hence $M(Y, 2, \mathcal{O}, d, H)$ is a the union of finitely many Maruyama's moduli schemes. A priori this number may grow to infinity as $d$ grows.

Theorem 0.1 will be proved in the unique section of this note. For similar statements on the number of components for $Y=\mathbf{P}^{3}$ and rank 2 vector bundles, see $[\mathbf{E}]$, and for $Y=\mathbf{P}^{5}$ and rank 3 vector bundles, see $[\mathbf{A O}]$. Here we will use in an essential way the rank 2 vector bundles on $\mathbf{P}^{3}$ constructed in $[\mathbf{E}]$ ("generalized null correlation bundles"). Our manifold $Y$ will have a morphism $\pi: Y \rightarrow \mathbf{P}^{3}$ and our bundles will be the pull-back bundles $\pi^{*}(E)$ 
with $E$ generalized null correlation bundle. The main points are to find a polarization $H$ on $Y$ (the same for all integers $d$ ) such that these bundles $\pi^{*}(E)$ are $H$-stable and to prove that these families are Zariski dense in their irreducible component of the moduli space $M(Y, 2, \mathcal{O}, d, H)$. A related construction was used in $[\mathbf{B M}]$.

The author was partially supported by MURST and GNSAGA of CNR (Italy). He thanks R.M. Miro-Roig for several nice discussions.

\section{Proof of Theorem 0.1 .}

Fix a very ample line bundle $A$ on $X$ such that $h^{i}\left(X, A^{\otimes t}\right)=0$ for all $(i, t)$ with either $i<n, t<0$ or $i>0$ and $t>0$. Let $M$ be the complete intersection of 4 general divisors in $|A|$ and let $Y$ be the blowing-up of $X$ along $M$. Any such $Y$ will be a birational model of $X$ satisfying the hypothesis of Theorem 0.1. Note that $h^{1}\left(Y, \mathcal{O}_{Y}\right)=h^{1}\left(X, \mathcal{O}_{X}\right)=0$. The 4 general divisors in $|A|$ cutting $M$ define a morphism $\pi: Y \rightarrow \mathbf{P}^{3}$. The morphism $\pi$ is flat because $\mathbf{P}^{3}$ and $Y$ smooth and each fiber of $\pi$ has dimension $n-3([\mathbf{H}]$, Ex. III.10.9). Our bundles on $Y$ will be the pull-backs by $\pi$ of the generalized null correlation bundles with trivial determinant introduced and studied in $[\mathbf{E}]$.

\section{Lemma 1.1.}

(a) We have $\pi_{*}\left(\mathcal{O}_{\mathbf{P}^{3}}\right) \cong \mathcal{O}_{\mathbf{P}^{3}}$.

(b) If $n \geq 5$, we have $R^{1} \pi_{*}\left(\mathcal{O}_{Y}\right)=0$. the same is true if $X=\mathbf{P}^{4}$ and $\operatorname{deg}(A)=1$ (i.e. $Y$ is a $\mathbf{P}^{1}$-bundle over $\mathbf{P}^{3}$ ).

Proof. Since the general fibers of $\pi$ are reduced and connected and $\pi$ is flat, part (a) is obvious. Now we will check part (b). Assume $n \geq 5$. We take $n-2$ general divisors $A_{i}, 1 \leq i \leq n-2$, in $|A|$ and we obtain $n-2$ exact sequences

$$
0 \rightarrow \mathcal{O}_{B}(-(x+1) A) \rightarrow \mathcal{O}_{B}(-x A) \rightarrow \mathcal{O}_{B \cap A^{\prime}}(-x A) \rightarrow 0
$$

with $B$ and $B \cap A^{\prime}$ complete intersection of divisors in $|A|$. Using Kodaira vanishing and the exact sequences (1) for all integers $x \leq 0$ we obtain the well-known fact that any complete intersection, $U$, of at most $n-2$ divisors of $|A|$ have $h^{0}\left(U, \mathcal{O}_{T}\right)=1, h^{i}\left(U, \mathcal{O}_{T}(-y A)\right)=0$ if $y<0$ and $i<\operatorname{dim}(U)$, and that $h^{1}\left(U, \mathcal{O}_{U}\right)=0$. In particular for every fiber $T$ of $\pi$ we have $h^{1}\left(T, \mathcal{O}_{T}\right)=0$. Since $\mathbf{P}^{3}$ is reduced by a base - change theorem for cohomology ([OSS, p. 11]) we obtain $R^{1} \pi_{*}\left(\mathcal{O}_{Y}\right)=0$. Now assume $X=\mathbf{P}^{4}$ and $\operatorname{deg}(A)=1$. Hence $Y \cong \mathbf{P}\left(\mathcal{O}_{\mathbf{P}^{3}} \oplus \mathcal{O}_{\mathbf{P}^{3}}(-1)\right)$ and $\pi$ is the projection as $\mathbf{P}^{1}$-bundle. Again $h^{1}\left(T, \mathcal{O}_{T}(1)\right)=0$ for every fiber of $\pi$ and we conclude in the same way. 
Corollary 1.2. Let $U$ be vector bundle $U$ on $\mathbf{P}^{3}$ and set $U^{\prime}:=\pi^{*}(U)$. We have $h^{1}\left(Y, U^{\prime}\right)=h^{1}\left(\mathbf{P}^{3}, U\right)$ and $h^{0}\left(Y, U^{\prime}\right)=h^{0}\left(\mathbf{P}^{3}, U\right)$.

Proof. Use 1.1, the projection formula and the Leray spectral sequence of $\pi$.

Corollary 1.3. For every vector bundle $E$ on $\mathbf{P}^{3}$ the tangent space to the deformation functor of $E$ on $\mathbf{P}^{3}$ has the same dimension as the tangent space of the deformation functor of $\pi^{*}(E)$ on $Y$. For any two stable vector bundles $E, E^{\prime}$ with the same rank on $\mathbf{P}^{3}$ the bundle $\pi^{*}(E)$ is isomorphic to $\pi^{*}\left(E^{\prime}\right)$ if and only if $E$ is isomorphic to $E^{\prime}$.

Proof. For the first part apply the first equality of 1.2 to the bundle $U:=$ $\operatorname{End}(E)$. For the second part apply the second equality of 1.2 to the bundle $U:=\operatorname{Hom}\left(E, E^{\prime}\right)$.

In $[\mathbf{E}]$ L. Ein constructed enough families of rank 2 vector bundles (called "generalized null correlation bundles") to prove the thesis of Theorem 0.1 taking $\mathbf{P}^{3}$ manifold $Y$. Each of these families were Zariski dense in a generically reduced component of the moduli scheme $M\left(\mathbf{P}^{3}, \mathcal{O}(1), \mathcal{O}, d\right)([\mathbf{E}$, Th. 2.2]). Thus by Corollary 1.3 to prove Theorem 0.1 on $Y$ it is sufficient to find a polarization $H$ on $Y$ such that all the bundles $\pi^{*}(E), E$ generalized null correlation bundle are $H$-stable. We will do this in two steps.

Step 1. Here we take as $Y$ the blowing-up $\sigma: Y \rightarrow X:=\mathbf{P}^{n}$ (any $n \geq 4$ ) of $\mathbf{P}^{n}$ along a linear subspace $M$ of dimension $n-4$, i.e. we take $X=\mathbf{P}^{n}$ and $\operatorname{deg}(A)=1$. The 4 hyperplanes defining $M$ induce a morphism $\pi: Y \rightarrow \mathbf{P}^{3}$. Let $D$ be the exceptional divisor of $\sigma$. Since the normal bundle of $M$ in $X$ is the direct sum of 4 line bundles of degree 1 we have $D \cong \mathbf{P}^{3} \times \mathbf{P}^{n-4}$ and $\pi \mid D$ (resp. $\sigma \mid D$ ) are the projection on the first (resp. second factor). If $n=4$, then $D \cong \mathbf{P}^{3}$ and the normal bundle of $D$ in $Y$ has degree -1 . We leave the case $n=4$ to the reader. From now on, we assume $n \geq 5$. We have $\operatorname{Pic}(D) \cong$ $\mathbf{Z}^{\oplus 2}$ and as generators of $\operatorname{Pic}(D)$ we take $\mathbf{O}(1,0):=(\pi \mid D)^{*}\left(\mathcal{O}_{\mathbf{P}^{3}}(1)\right)$ and $\mathbf{O}(0,1):=(\sigma \mid D)^{*}\left(\mathcal{O}_{\mathbf{P}^{n-4}}(1)\right)$. We have $\operatorname{Pic}(Y) \cong \mathbf{Z}^{\oplus 2}$ and the restriction $\operatorname{map} \operatorname{Pic}(Y) \rightarrow \operatorname{Pic}(D)$ is bijective. Set $O(1,0):=\pi^{*}\left(\mathcal{O}_{\mathbf{P}^{3}}(1)\right)$ and $O(0,1):=$ $\sigma^{*}\left(\mathcal{O}_{X}(1)\right)$. Hence for all integers $a, b$ we have $O(a, b) \mid D \cong \mathbf{O}(a, b)$. Fix a generalized null correlation bundle $E$ on $\mathbf{P}^{3}$ and set $F:=\pi^{*}(E)$. Let $M:=$ $O(x, y)$ be a saturated line bundle contained in $F$. Restricting $M \mid D$ and $E$ to a general fiber of $\pi$ we obtain $x \leq 0$. Since $E$ is stable, restricting $M \mid D$ and $F \mid D$ to a general fiber of $\sigma \mid D$ we obtain $y \leq 0$. Fix an integer $b$ with $0 \leq b \leq n-1$. Since $D$ is a product of projective spaces, the product of $b$ copies of $\mathbf{O}(1,0)$ and $n-1-b$ copies of $\mathbf{O}(0,1)$ in the Chow ring $A(D)$ of $D$ is 0 if $b \neq 3$ and 1 if $b=3$. Fix $H \in \operatorname{Pic}(Y), H=O(u, v)$ with $u>0$ 
and $v>0$, and ample. Since both $O(1,0)$ and $O(0,1)$ are effective, we have $H^{n-1} \cdot M<0$. Hence $F$ is $H$-stable in the sense of Mumford-Takemoto.

Step 2. Now we consider the general case (here $n \geq 5$ ). We take a general $n$-dimensional projective subspace $|V| \subseteq|A|$ containing the $n-4$ divisors defining $M$. We may assume $|V|$ base point free and hence defining a morphism $f: X \rightarrow \mathbf{P}^{n}$ such that the image of $M$ is a linear subspace $M^{\prime}$ of dimension $n-4$. Call $Y^{\prime}$ the blowing-up of $\mathbf{P}^{n}$ along $M^{\prime}$ and apply Step 1 to $Y^{\prime}$. By construction we have a finite map $g: Y \rightarrow Y^{\prime}$ and we will take $H:=g^{*}\left(H^{\prime}\right)$ as polarization on $Y$ with $H^{\prime}$ any polarization on $Y^{\prime}$ used in Step 1. We have to check that if $F$ is a rank $2 H^{\prime}$-stable bundle on $Y^{\prime}$, then $g^{*}(F)$ is $H$-stable. Let $u: C \rightarrow B$ be a finite map of smooth projective curves and $G$ a rank 2 stable vector bundle on $B$. Assume that $u$ does not factor through an étale cover of degree $>1$. Then, assuming characteristic $0, u^{*}(G)$ is stable $([\mathbf{L}$, Remark 2.3]). Taking as $B$ a general complete intersection curve of divisors in $\mathbf{P}\left(H^{0}\left(Y^{\prime}, H^{\prime, \otimes t}\right)\right)$ with $t$ large and as $u$ the restriction of $g$, we conclude if this restriction map does not factor through an étale cover of degree $>1$. Taking $A$ sufficiently ample and $|V|$ general we may assume that $g: Y \rightarrow Y^{\prime}$ has irreducible ramification locus $R$ and that for a general $x \in R$ we have $\operatorname{card}\left(g^{-1}(x)\right)=\operatorname{deg}(g)-1$. In this way we reduce to a case in which the monodromy group of $u: C \rightarrow B$ is transitive and generated by simple transpositions, i.e. it is the full symmetric group $S_{\operatorname{deg}(u)}$. Hence $u$ does not factor non trivially and we conclude the proof of Theorem 0.1.

\section{References}

[AO] V. Ancona and G. Ottaviani, The Harrocks bundles of rank 3 on $\mathbf{P}^{5}$, J. reine angew Math., 460 (1995), 69-92.

[BM] E. Ballico and R.M. Miró-Roig, A lower bound for the number of components of the moduli schemes of stable rank 2 vector bundles on projective 3-folds, preprint.

[E] L. Ein, Generalized null correlations bundles, Nagoya Math., 111 (1988), 13-24.

[H] R. Hartshorne, Algebraic geometry, Graduate text in Math., 52, Springer-Verlag, 1977.

[L] H. Lange, On stable and ample vector bundles of rank 2 on curves, Math. Ann., 238 (1978), 193-202.

[OSS] C. Okonek, M. Schneider and H. Splinder, Vector bundles on projective spaces, Progress in Math., 3 Birkhäuser, 1980.

Received August 15, 1996.

UNIVERSITY OF TRENTO

38050 Povo (TN)

ITALY

E-mail address: ballico@science.unitn.it 